\title{
Coenzyme Q10 Supplementation Effects on In Vitro Maturation, Fertilization, and Early Embryonic Development in Pigs
}

\author{
CAITLIN STREACKER and BRIAN D. WHITAKER', University of Findlay, Findlay, OH, USA.
}

ABSTRACT. The objective of this study was to determine the reduction of polyspermic penetration, and increase of mitochondrial activity, in early pig embryonic development by supplementing different concentrations of coenzyme Q10 during oocyte maturation. Oocytes $(n=1,100)$ were supplemented during the last $24 \mathrm{~h}$ of maturation with 0 (control), 10, 50, or $100 \mu \mathrm{M}$ of coenzyme Q10. After in vitro fertilization (IVF), embryos were evaluated for fertilization kinetics (penetration, polyspermic penetration, male pronuclear formation), and subsequent embryonic development and mitochondrial activity. Supplementation of $100 \mu \mathrm{M}$ coenzyme Q10 was detrimental to the oocytes, as they had significantly lower $(p<0.05)$ fertilization kinetic and early embryonic development rates to the other treatment groups. There were no differences in fertilization kinetic and early embryonic development rates between the 0, 10 and $50 \mu \mathrm{M}$ coenzyme $\mathrm{Q} 10$ treatment groups. Oocytes, matured in medium supplemented with $50 \mu \mathrm{M}$ coenzyme Q10, ultimately developed into embryos with a significantly greater $(p<0.05)$ presence of intact mitochondrial membranes (observed at both 48 and $144 \mathrm{~h}$ post-IVF) compared to oocytes not supplemented with coenzyme Q10. In summary, supplementation of $100 \mu \mathrm{M}$ coenzyme Q10 during oocyte maturation is detrimental, yet supplementation of $50 \mu \mathrm{M}$ coenzyme Q10 leads to a higher occurrence of intact mitochondrial membranes in the in vitro produced pig embryos.

\section{INTRODUCTION}

Pig embryos produced from in vitro fertilization (IVF) are an ideal biomedical research model due to the similar genetics, organ development, and disease progression as seen in humans (Whyte and Prather 2011). In vitro fertilization in pigs (Sus scrofa) has been as little as one-half as efficient compared to other species such as cows or sheep (Mtango et al. 2002). There are several contributing factors to inefficient IVF including lack of oocyte cytoplasmic maturation, high rate of polyspermic penetration (polyspermy), and insufficient blastocyst formation (Niemann and Rath 2001). Therefore, in vitro techniques in pigs need to be improved in order to increase the efficiency and success of in vitro derived embryos.

Changing the in vitro maturation conditions can improve IVF success and increase efficiency in producing pig embryos (Grupen 2014). Oxidative stress is a major contributing factor to both the lack of cytoplasmic maturation and to an increase in reactive oxygen species (ROS) development. Oxidative stress has also been implicated in causing protein and DNA breakdown, resulting in apoptosis (Whitaker and Knight 2008). Reducing detrimental levels of ROS and decreasing oxidative stress can promote oocyte development and embryo survivability.

${ }^{1}$ Address correspondence to Brian D. Whitaker, University of Findlay, 1000 North Main Street, Findlay, OH 45840.

Email: whitaker@findlay.edu
Antioxidants reduce oxidative stress and are attractive additives to a maturation media to promote cytoplasmic maturation and blastocyst development. As an example, Tatemoto et al. (2001) observed this effect by supplementing their maturation media with the antioxidant ascorbic acid 2-O- $\alpha$-glucoside, which reduced the ROS and improved embryonic development.

Coenzyme Q10 (also known as ubiquinone) is an antioxidant that has been used as a supplement (30 and $100 \mu \mathrm{M}$ ) to the IVF media in cows, resulting in increased blastocyst formation and ATP production (Stojikovic et al. 1999). Coenzyme Q10 is also an integral component for the production of ATP in the electron transport chain (ETC) and is imbedded in the lipid core of the mitochondrial membrane (Turunen et al. 2004). In the ETC, coenzyme Q10 acts as an electron acceptor for electrons from NADH in complex I and from succinate in complex II. In complex III of the ETC, cytochrome c accepts electrons from coenzyme Q. Electrons continue to flow through complex IV where they are used to generate ATP from ADP (McKee and McKee 2012). Coenzyme Q10 acts as a cofactor for uncoupling proteins to translocate hydrogen

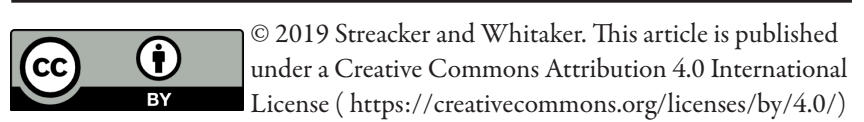


ions into the mitochondria to establish a proton gradient for oxidative phosphorylation to produce ATP (Turunen et al. 2004). Oxidative stress can also result when unpaired electrons are released from the ETC.

The synthesis of ATP is disrupted when a mitochondrial permeability transition pore (PTP) expands which results in mitochondrial membrane depolarization, ATP depletion, and ultimately cellular death. Coenzyme Q10 prevents the opening of the PTP and thus facilitates an adequate supply of ATP (Papucci et al. 2003).

No reports could be located concerning the supplementation of coenzyme Q10 to pig oocyte maturation media. The objective of this study was to reduce polyspermic penetration, and improve both male pronuclear (MPN) formation and early embryonic mitochondrial function, by supplementating 10, 50, or $100 \mu M$ coenzyme Q10 intopigoocyte maturation media. Following oocyte maturation and IVF, zygotes were evaluated for penetration, polyspermic penetration, and MPN formation to determine IVF effectiveness. Embryos were evaluated for cleavage, blastocyst formation, and mitochondrial membrane potential.

\section{Media}

\section{METHODS AND MATERIALS}

Unless otherwise stated, all chemicals were purchased from Sigma-Aldrich ${ }^{\circledR}$ Inc. (St. Louis, Missouri, USA). The oocyte maturation medium was Medium 199 (M199) with Earle's salts (Fisher Scientific ${ }^{\circledR}$ Co. LLC, Pittsburgh, Pennsylvania, USA) supplemented with $5 \mu \mathrm{g} / \mathrm{ml}$ follicle stimulating hormone (FSH), $1 \mu \mathrm{l} / \mathrm{ml}$ insulin, $50 \mathrm{ng} / \mathrm{ml}$ gentamicin sulfate, $10 \mathrm{ng} / \mathrm{ml}$ epidermal growth factor, and $10 \%$ fetal calf serum (v/v; FCS). The IVF medium was a modified Tris-buffered media as originally developed by Abeydeera and Day (1997). The embryo culture medium was North Carolina State University (NCSU)-23 medium (Petters and Wells 1993$)$ containing $0.4 \%(\mathrm{w} / \mathrm{v})$ bovine serum albumin (BSA).

All media were filtered through a $0.22 \mu \mathrm{m}$ pore mixed cellulose esters membrane syringe filter (Fisher Scientific ${ }^{\circledR}$ Co. LLC, Pittsburgh, Pennsylvania, USA). All incubations were carried out in media droplets under mineral oil at $38.5^{\circ} \mathrm{C}$ in a humid atmosphere of $5 \% \mathrm{CO}_{2}$ unless otherwise indicated.

\section{Maturation of Oocytes}

Oocytes ( $\mathrm{n}=1,350$; Applied Reproductive Technology LLC, Monona, Wisconsin, USA) were aspirated from mature follicles (3 to 6 $\mathrm{mm}$ diameter) obtained and pooled from adult crossbred sows (at least 18 months of age) at an abattoir. The average elapsed time between ovary collection and follicular aspiration was $5 \mathrm{~h}$. The first phase of maturation (from 0 to $22 \mathrm{~h}$ ) began when the oocytes were shipped overnight, in oocyte maturation media at $38.5^{\circ} \mathrm{C}$, to our laboratory. Only oocytes observed with uniform ooplasm and compact cumulus cells $22 \mathrm{~h}$ after initial placement in oocyte maturation medium by the supplier were selected using a micropipette.

The second phase of maturation (from 22 to 46 h) began when selected oocytes $(n=1,100)$ were washed 3 times in oocyte maturation medium and placed (50-oocytes per well) in $500 \mu \mathrm{L}$ of oocyte maturation medium without FSH, insulin, and FCS. At this point - the start of this second phase of maturation - the oocytes were divided in to 4 treatment groups; a different concentration $(0,10,50$, or $100 \mu \mathrm{M})$ of coenzyme Q10 was supplemented to the medium of each group. After $24 \mathrm{~h}$ of additional incubation, cumulus cells were removed from the oocytes by repeat pipetting in M199 containing $0.1 \%(\mathrm{w} / \mathrm{v})$ hyaluronidase. Oocytes with cumulus cells removed, but observed to have uniform ooplasm, were immediately washed in IVF media and used for further analysis as described below.

\section{In Vitro Fertilization and Embryo Culture}

Approximately $1 \mathrm{~h}$ before the completion of oocyte maturation, a frozen semen pellet (International Boar Semen, Eldora, Iowa, USA) was thawed in IVF medium. The semen was then centrifuged at $36.3 \mathrm{~g}$ for $5 \mathrm{~min}$, washed in IVF medium, and again centrifuged twice at $553 \mathrm{~g}$ for $5 \mathrm{~min}$. After washing, the sperm pellet was resuspended in IVF medium to a concentration of $4 \times 10^{5}$ spermatozoa per $\mathrm{mL}$. A volume of $50 \mu \mathrm{L}$ of this IVF medium (with the spermatozoa) was added to groups of 30 oocytes. The final concentration was approximately 65 spermatozoa per oocyte. After 4 to $6 \mathrm{~h}$ of IVF, the zygotes were washed 3 times with NCSU-23 medium and placed (in groups of 50) into $100 \mu \mathrm{L}$ of NCSU-23 medium for culture. 


\section{Experimental Design}

Experiment 1: effects of different doses of coenzyme $Q 10$ on embryo production.

This experiment studied the effects of supplementing $0,10,50$, or $100 \mu \mathrm{M}$ coenzyme Q10 to maturation media during the second phase of oocyte maturation (the period from 22 to $46 \mathrm{~h}$ after follicular aspiration, but before IVF).

The first part of Experiment 1 examined and recorded initial fertilization kinetics. Approximately $12 \mathrm{~h}$ after the completion of IVF, the zygotes $(\mathrm{n}=400$; 100 per treatment group, times the 4 treatment groups) were removed from the NCSU-23 medium and mounted and fixed with $25 \%$ acetic acid in ethanol $(\mathrm{v} / \mathrm{v})$ at room temperature. After $48 \mathrm{~h}$ of fixation, the zygotes were stained with $1 \%$ orcein $(w / v)$ in $45 \%$ acetic acid (v/v) and examined using a phasecontrast microscope at $400 \times$ magnification. Endpoints measured were the percent of oocytes penetrated, the percent of polyspermic oocytes, and the percent of oocytes penetrated with MPN formation. Oocytes were considered penetrated when they had one or more swollen sperm head(s) or a MPN formed and their corresponding sperm tails.

The second part of Experiment 1 examined subsequent embryonic development. Embryos $(n=400 ; 100$ per treatment group, times the 4 treatment groups) in NCSU-23 medium were observed - under a stereomicroscope-for the percent of embryos cleaved $48 \mathrm{~h}$ after the completion of IVF and blastocysts developed at $144 \mathrm{~h}$ after the completion of IVF.

\section{Experiment 2: test of the effects of coenzyme Q10 on embryonic mitochondrial activity.}

Experiment 2 applied the results of Experiment

1. Experiment 1 determined the most advantageous concentration of coenzyme Q10 which (when supplemented to the oocyte maturation media during the second phase of oocyte maturation) elicited the lowest rate of polyspermic penetration and highest rates of penetration, MPN formation, cleavage, and blastocyst formation. This optimal concentration of coenzyme Q10, ultimately determined to be $50 \mu \mathrm{M}$, was used exclusively for Experiment 2.

Mitochondrial membranes must remain intact to ensure proper functionality throughout embryonic development. In Experiment 2, mitochondrial activity was measured-as described below_-by determining the ratios of embryos that displayed red fluorescence in the mitochondrial matrix (indicating intact, proper functioning, membranes) versus embryos that had green fluorescence (indicating damaged/ disrupted membranes) dispersed throughout the cell. This measurement, quantifying mitochondrial functionality, was taken at 12,48 , and $144 \mathrm{~h}$ after the completion of IVF.

Oocytes used in Experiment 2 ( $\mathrm{n}=300$ total as follows: a coenzyme Q10 treatment group of $n=150$; 50 per time interval, times 3 intervals. Plus an untreated control group of $\mathrm{n}=150 ; 50$ per time interval, times 3 intervals) were incubated in maturation media supplemented with $50 \mu \mathrm{M}$ coenzyme Q10 during the second phase of oocyte maturation (the period from 22 to $46 \mathrm{~h}$ after follicular aspiration), this was followed by IVF and culture in NCSU-23 medium.

At 12,48 , and $144 \mathrm{~h}$ after the completion of IVF, embryos were removed from the NCSU-23 medium, stained with 5, 5',6,6'-tetrachloro-1, 1',3,3'tetraethylbenzimidazolocarbocyanine iodide (JC-1; Sigma-Aldrich ${ }^{\circledR}$ Inc.), and mounted onto slides. Embryos were examined using fluorescent microscopy (excitation maximum wavelength $=525 \mathrm{~nm}$ and emission maximum wavelength $=590 \mathrm{~nm}$ ). Images

Table

Effects of coenzyme Q10 on oocyte fertilization kinetics $12 \mathrm{~h}$ after completion of IVF

\begin{tabular}{llll}
\hline \hline Treatment group $^{\dagger}$ & Oocytes penetrated (\%) & Polyspermic oocytes $^{\S}(\%)$ & Oocytes with MPN $^{\S}(\%)$ \\
\hline No coenzyme Q10 & $86.00 \pm 4.97^{\mathrm{a}}$ & $26.67 \pm 6.35^{\mathrm{a}}$ & $46.51 \pm 9.54^{\mathrm{a}}$ \\
$10 \mu \mathrm{M}$ coenzyme Q10 & $80.00 \pm 9.43^{\mathrm{a}}$ & $26.25 \pm 3.65^{\mathrm{a}}$ & $53.75 \pm 4.33^{\mathrm{a}}$ \\
$50 \mu \mathrm{M}$ coenzyme Q10 & $95.00 \pm 7.14^{\mathrm{a}}$ & $33.68 \pm 8.32^{\mathrm{a}}$ & $57.89 \pm 4.54^{\mathrm{a}}$ \\
$100 \mu \mathrm{M}$ coenzyme Q10 & $35.00 \pm 4.94^{\mathrm{b}}$ & $5.71 \pm 10.35^{\mathrm{b}}$ & $5.71 \pm 11.85^{\mathrm{b}}$ \\
\hline
\end{tabular}

Treatment groups were the concentration of coenzyme Q10 supplemented to the oocyte maturation media in the second phase of maturation from 22 to $46 \mathrm{~h}$ ( $\mathrm{n}=400 ; 100$ per treatment group) in Experiment 1.

$\$$ Percentage of the number of oocytes penetrated.

${ }^{\mathrm{a}, \mathrm{b}}$ Data are expressed as least-squares mean \pm SEM. Means with different superscripts, and within the same column, differ significantly $(\mathrm{p}<0.05)$. Significant differences between columns are not comparable. 
were recorded digitally and the fluorescence brightness at the equatorial section of each embryo (stained by JC1) was calculated using computer software (Nikon ${ }^{\circledR}$ NIS-Elements; Nikon Instruments Inc., Melville, New York, USA). The data were presented as the ratio (red to green) of relative fluorescence intensity.

\section{Statistical Analysis}

Data were analyzed by one-way ANOVA using the PROC GLM features of the GLM procedure in SAS/STAT ${ }^{\circledR}$ software (SAS Institute Inc., Cary, North Carolina, USA). When there was a significant effect, significant differences were determined using the LSMEANS statement and TUKEY adjustment for multiple comparisons. The effects included in the initial model were treatment, well, and replicate. Well and replicate effects were not significant $(\mathrm{p}>0.05)$ and were deleted from the final models. A chi-square test was used to determine percentages of embryos reaching the different developmental stages for each treatment. In all analyses, $\mathrm{p}<0.05$ was considered to be significant. Results are expressed as the least-squares mean \pm the standard error of the mean (SEM).

\section{Experiment 1}

\section{RESULTS}

Measured approximately $12 \mathrm{~h}$ after completion of IVF, oocytes supplemented with $100 \mu \mathrm{M}$ coenzyme Q10 had significantly lower $(\mathrm{p}<0.05)$ incidences of penetration $(35.00 \pm 4.94 \%)$, polyspermy $(5.71 \pm 10.35 \%)$, and MPN formation $(5.71 \pm 11.85 \%)$ compared to all other treatment

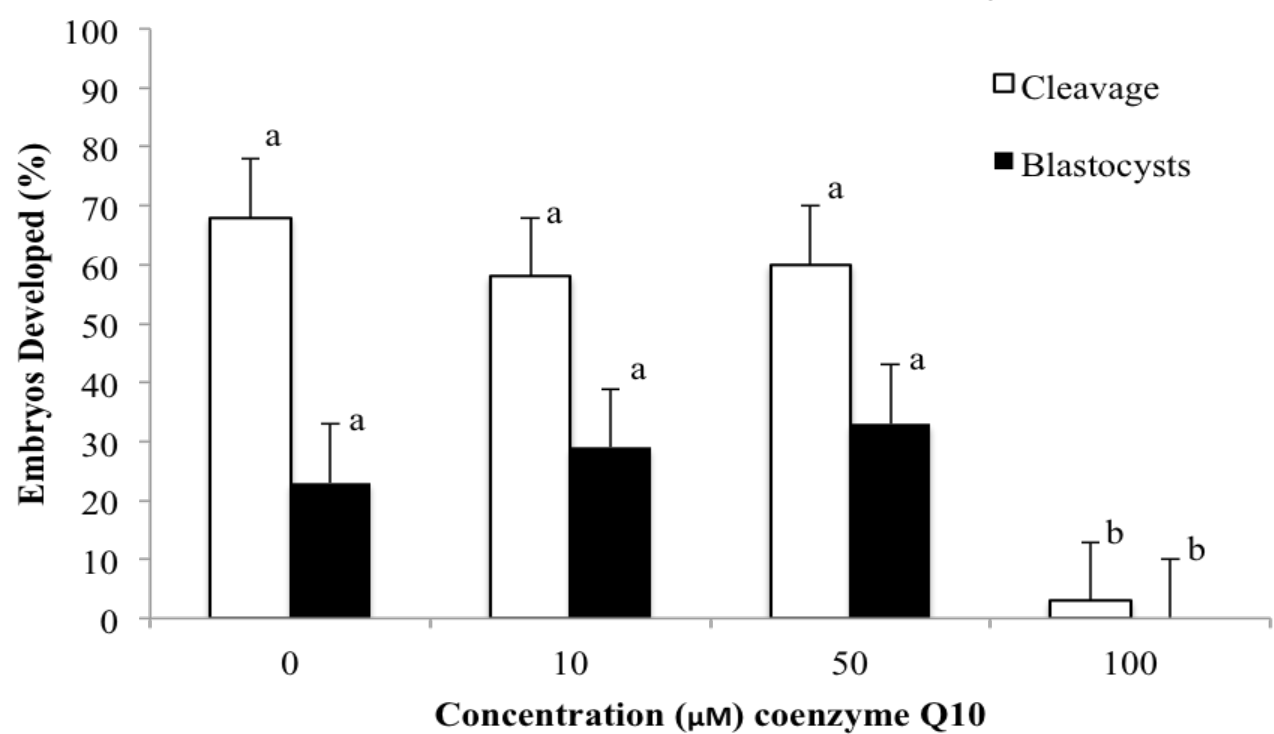

FIGURE 1. Effects of different coenzyme Q10 concentrations (supplemented in the oocyte maturation media in the second phase of maturation from 22 to $46 \mathrm{~h}$ ) on embryo development $(n=400)$ in Experiment 1. Data expressed as least-squares mean \pm SEM. Cleavage observed $48 \mathrm{~h}$ after completion of IVF; blastocysts developed $144 \mathrm{~h}$ after completion of IVF. Significant differences between cleavage and blastocyst data are not comparable. ${ }^{a, b}$ Means with different superscripts differ significantly $(p<0.05)$. groups (Table). There were no statistical differences between the other treatment groups for all IVF endpoints considered.

Embryo development results are shown in Fig. 1. The 0,10 , and $50 \mu \mathrm{M}$ coenzyme Q10 treatment groups showed no statistical differences in the percentage of embryos cleaved by $48 \mathrm{~h}$ after IVF or percentage of blastocysts formed by $144 \mathrm{~h}$ after IVF. However, the oocytes supplemented with $100 \mu \mathrm{M}$ coenzyme Q10 had a significantly lower $(\mathrm{p}<0.05)$ percentage of embryos cleaving by $48 \mathrm{~h}$ after IVF $(3.00 \pm 19.06 \%)$ and no oocytes reached the blastocysts stage of development by $144 \mathrm{~h}$ after IVF.

\section{Experiment 2}

Supplementation of $50 \mu \mathrm{M}$ of coenzyme Q10 was the highest level that did not have detrimental effects on sperm penetration, MPN formation, and cleavage and blastocyst formation-and thus was the supplementation level used to determine mitochondrial activity. Supplementation of $50 \mu \mathrm{M}$ coenzyme Q10 resulted in a significantly higher ratio $(p<0.05)$ of intact mitochondrial matrixes (fluorescing red) to dispersed mitochondrial matrixes (causing the whole cell to fluoresce green) as compared to the control (0 $\mu \mathrm{M}$ coenzyme Q10 supplementation). This was observed at both $48 \mathrm{~h}$ post-IVF ( 0.51 vs. 0.45 relative fluorescence intensity, $50 \mu \mathrm{M}$ vs. $0 \mu \mathrm{M}$ respectively) and $144 \mathrm{~h}$ post-IVF $(0.49 \mathrm{vs} .0 .42$ relative fluorescence intensity, $50 \mu \mathrm{M}$ vs. $0 \mu \mathrm{M}$ respectively) (Fig. 2). There was no significant difference in mitochondrial activity between treatment groups at $12 \mathrm{~h}$ post-IVF. 


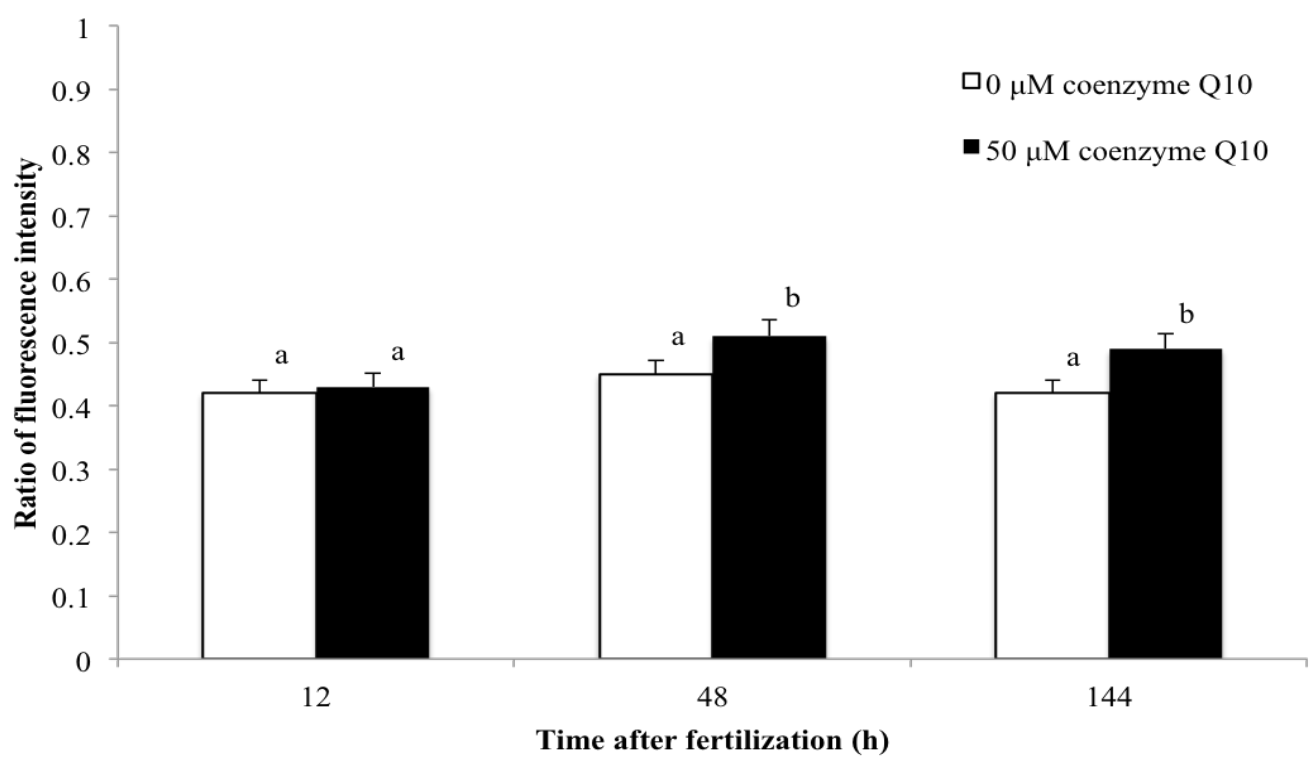

FIGURE 2. Effect of $50 \mu \mathrm{M}$ coenzyme Q10 (supplemented in the oocyte maturation media in the second phase of maturation from 22 to $46 \mathrm{~h}$ ) on embryo mitochondrial activity $(\mathrm{n}=300)$ at 12,48 , and $144 \mathrm{~h}$ after IVF in Experiment 2. Data, expressed as least-squares mean \pm SEM, show the ratio of red florescent intensity (intact mitochondria) to green florescent intensity (dispersed mitochondria) in the stain color of JC-1. There were no significant differences in the ratio of fluorescence intensity at $12 \mathrm{~h}$ post-IVF.

a,b Means with different superscripts, and within the same time interval, differ significantly $(p<0.05)$.

\section{DISCUSSION}

Coenzyme Q10 is located in the cytoplasm and mitochondrial membranes that carry hydrogen ions and electrons. It plays a role in cell growth, energy metabolism, and preventing oxidative damage (Crane et al. 1993). During the production of in vitro derived pig embryos, the embryo culture step is the longest step and fails to mimic the in vivo environments of the oviduct and uterus. Pig embryos produced in vitro continually have lesser developmental competence-including lower cleavage and blastocyst development rates-than embryos cultured in vivo (Gil et al. 2010). Previous studies have shown that culture media containing lower levels of ATP and glucose during the early stages of embryonic development improve success rates (Abeydeera 2002; Kikuchi et al. 2002).

Reducing oxygen tension during culture could reduce the oxidative stress placed on the embryos; however, there are conflicting reports on the optimal levels of oxygen in the system (Machaty et al. 1998; Karja et al. 2004; Yoshioka et al. 2008). Stojkovic et al. (1999) reported that $30 \mu \mathrm{M}$ or $100 \mu \mathrm{M}$ coenzyme Q10 supplementation during in vitro embryo culture in cows improves cleavage, blastocyst, and hatching blastocyst rates. Stojkovic et al. (1999) also reported that supplementation with coenzyme Q10 also increased the number of embryonic cells and ATP content per cell. In partial agreement with these studies, the results of this study indicate that supplementation of the maturation media with $50 \mu \mathrm{M}$ coenzyme Q10 increases the ratio of intact mitochondrial matrixes during embryonic development in pigs.

Results of the current study indicated that supplementation of oocyte maturation media with $100 \mu \mathrm{M}$ coenzyme Q10 was detrimental to the oocyte and resulted in death. Maturation media supplemented with $50 \mu \mathrm{M}$ coenzyme Q10 did not have significant effects on oocyte fertilization kinetics (sperm penetration, polyspermic penetration, or MPN formation) compared to the control. Therefore, $50 \mu \mathrm{M}$ coenzyme Q 10 was chosen as the appropriate level of supplementation for the mitochondrial study. These findings were expected: coenzyme Q10 is located in plasma and mitochondrial membranes and would not have an immediate impact or function during oocyte maturation or fertilization. Coenzyme Q10 potentially could be acting as an antioxidant in the media during oocyte maturation (Frei et al. 1990), however these effects were not considered in the current study.

Embryos, developed from oocytes incubated in maturation media supplemented with 50 $\mu \mathrm{M}$ coenzyme Q10 (during the second phase of maturation), did show significant improvement in mitochondrial activity. At both 48 and 144 h after IVF the embryos sourced from the $50 \mu \mathrm{M}$ coenzyme Q10matured oocytes displayed a significantly higher ratio $(\mathrm{p}<0.05)$ of intact mitochondrial matrixes compared 
to dispersed mitochondrial matrixes (as compared to the control). This is similar to two previous findings: First, coenzyme Q10 supplementation improves embryonic development beyond the cleavage stage - most likely due to mitochondrial regulation (Hwang et al. 2016). Second, antioxidants could reduce ROS that would induce PTP openings in the mitochondrial membrane (Vianello et al. 2012).

Although there have been advances in the in vitro production of pig embryos, the success rate is still below the corresponding rates in vivo. Suboptimal defined culture conditions create a stressful environment for developing embryos in vitro and inhibit their ability to adequately progress to a hatched blastocyst (Gil et al. 2010).

During early embryonic development, mitochondria are essential in providing enough energy for the cell to function properly. High concentrations of ATP are required for perinuclear mitochondria clustering for cleavage and then homogenous distribution in a blastocyst (Sun et al. 2001). Additionally, the plasma membrane ion transport system found in the mitochondria depends on coenzyme Q10 to carry electrons and, in the form of ubiquinol, scavenge free radicals to alleviate peroxidative damage. Increasing the availability of coenzyme Q10 during oocyte maturation could increase its incorporation into the mitochondria during subsequent embryonic development and act as an antioxidant in the environment. At this point, however, it is uncertain which mechanism of action the coenzyme Q10 supplementation is affecting; a topic which warrants further studies.

\section{CONCLUSION}

This is the first study that considers the effects of separately supplementing 4 different concentrations $(0,10,50$, and $100 \mu \mathrm{M})$ of coenzyme Q10 into the maturation media of pig oocytes intended to subsequently undergo IVF and embryo culture. Test oocytes, initially incubated in maturation media supplemented with $50 \mu \mathrm{M}$ coenzyme Q10, later experienced similar IVF kinetic, cleavage, and blastocyst success rates-but ultimately yielded embryos with significantly higher proportions of intact functioning mitochondrial membranes (at both 48 and $144 \mathrm{~h}$ post-IVF)_than the control oocytes. These findings may improve success rates for producing in vitro derived pig embryos.

\section{LITERATURE CITED}

Abeydeera LR, Day BN. 1997. In vitro penetration of pig oocytes in a modified Tris-buffered medium: effect of BSA, caffeine and calcium. Theriogenology. 48(4):537-544. https://doi.org/10.1016/S0093-691X(97)00270-7

Abeydeera LR. 2002. In vitro production of embryos in swine. Theriogenology. 57(1):257-273. https://doi.org/10.1016/S0093-691X(01)00670-7

Crane FL, Sun IL, Sun EE. 1993. The essential functions of coenzyme Q. Clin Investigator. 71(Suppl 8):S55-S59. https://doi.org/10.1007/BF00226841

Grupen CG. 2014. The evolution of porcine embryo in vitro production. Theriogenology. 81(1):24-37. https://doi.org/10.1016/j.theriogenology.2013.09.022

Frei B, Kim MC, Ames BN. 1990. Ubiquinol-10 is an effective lipid-soluble antioxidant at physiological concentrations. P Natl Acad Sci USA. 87(12):4879-4883. https://doi.org/10.1073/pnas.87.12.4879

Gil MA, Cuello C, Parrilla I, Vazquez JM, Roca J, Martinez EA. 2010. Advances in swine in vitro embryo production technologies. Reprod Domest Anim. 45(2):40-48. https://doi.org/10.1111/j.1439-0531.2010.01623.x

Hwang IS, Kwon DJ, Kwak TU, Lee JW, Im GS, Hwang S. 2016. Improved survival and developmental rates in vitrifiedwarmed pig oocytes after recovery culture with coenzyme Q10. Cryoletters. 37(1):59-67.

Karja NWK, Wongsrikeao P, Murakami M, Agung B, Fahrudin M, Nagai T, Otoi T. 2004. Effect of oxygen tension on the development and quality of porcine in vitro fertilized embryos. Theriogenology. 62(9):1585-1595. https://doi.org/10.1016/j.theriogenology.2004.03.012

Kikuchi K, Onishi A, Kashiwazaki N, Iwamoto M, Noguchi J, Kaneko H, Anita T, Nagai T. 2002. Successful piglet production after transfer of blastocysts produced by a modified in vitro system. Biol Reprod. 66(4):1033-1041. https://doi.org/10.1095/biolreprod66.4.1033

Machaty Z, Day BN, Prather RS. 1998. Development of early porcine embryos in vitro and in vivo. Biol Reprod. 59(2):451455. https://doi.org/10.1095/biolreprod59.2.451

McKee T, McKee JR. 2012. Biochemistry: the molecular basis of life. 5th ed. New York (NY): Oxford University Press.

Mtango NR, Varisanga MD, Juan DY, Wongrisekeao P, Suzuki T. 2002. Development to blastocyst stage of pig oocytes matured, fertilized and electroactivated in vitro. Arch Tierzucht. 45:547556. https://doi.org/10.5194/aab-45-547-2002

Niemann H, Rath D. 2001. Progress in reproductive biotechnology in swine. Theriogenology. 56(8):1291-1304. https://doi.org/10.1016/S0093-691X(01)00630-6

Papucci L, Schiavone N, Witort E, Donmini M, Lapucci A, Tempstini A, Formigli L, Zecchi-Orlandini S, Orlandini G, Carella G, Brancto R, Capaccioli S. 2003. Coenzyme Q10 prevents apoptosis by inhibiting mitochondrial depolarization independently of its free radical scavenging property. J Biol Chem. 278(30):28220-28228.

https://doi.org/10.1074/jbc.M302297200 
Petters RM, Wells KD. 1993. Culture of pig embryos. J Rep Fer S. 48:61-73.

Stojkovic M, Westesen K, Zakhartchenko V, Stojkovic P, Boxhammer K, WolfE. 1999. Coenzyme Q10 in submicronsized dispersion improves development, hatching, cell proliferation, and adenosine triphosphate content of in vitro-produced bovine embryos. Biol Reprod. 61(2):541-547. https://doi.org/10.1095/biolreprod61.2.541

Sun QY, Wu GM, Lai L, Park KW, Cabot R, Cheong HT, Day BN, Prather RS, Schatten H. 2001. Translocation of active mitochondria during pig oocyte maturation, fertilization and early embryo development in vitro. Reproduction. 122(1):155-163.

https://rep.bioscientifica.com/view/journals/rep/122/1/155.xml

Tatemoto H, Ootaki K, Shigeta K, Muto N. 2001. Enhancement of developmental competence after in vitro fertilization of porcine oocytes by treatment with ascorbic acid $2-\mathrm{O}-\alpha-$ glucoside during in vitro maturation. Biol Reprod. 65(6):18001806. https://doi.org/10.1095/biolreprod65.6.1800
Turunen M, Olsson J, Dallner G. 2004. Metabolism and function of coenzyme Q. BBA-Biomembranes. 1660(1-2):171-199. https://doi.org/10.1016/j.bbamem.2003.11.012

Vianello A, Casolo V, Petrussa E, Peresson C, Patui S, Bertolini A, Passamonti S, BraidotE, Zancani M. 2012. The mitochondrial permeability transition pore (PTP)—an example of multiple molecular exaptation? BBA-Bioenergetics. 1817(11):20722086. https://doi.org/10.1016/j.bbabio.2012.06.620

Whitaker BD, Knight JW. 2008. Mechanisms of oxidative stress in porcine oocytes and the role of anti-oxidants. Reprod Fert Develop. 20(6):694-702. https://doi.org/10.1071/RD08037

Whyte JJ, Prather RS. 2011. Genetic modifications of pigs for medicine and agriculture. Mol Reprod Dev. 78(10-11):879891. https://doi.org/10.1002/mrd.21333

Yoshioka K, Suzuki C, Onishi A. 2008. Defined system for in vitro production of porcine embryos using a single basic medium. J Reprod Develop. 54(3):208-213.

https://doi.org/10.1262/jrd.20001 